\title{
miR-223-3p inhibits the progression of atherosclerosis via down- regulating the activation of MEK1/ERK1/2 in macrophages
}

\author{
Daofeng You ${ }^{1}$, Qiuge Qiao ${ }^{2}$, Katsushige Ono ${ }^{3}$, Mei Wei ${ }^{1}$, Wenyun Tan ${ }^{1}$, Cuihua Wang ${ }^{1}$, \\ Yangong Liu', Gang Liu' ${ }^{1}$, Mingqi Zheng ${ }^{1}$ \\ ${ }^{1}$ Hebei Medical University First Affiliated Hospital, Shijiazhuang 050023, Hebei, China \\ ${ }^{2}$ Hebei Medical University Second Affiliated Hospital, Shijiazhuang 050023, Hebei, China \\ ${ }^{3}$ Department of Pathophysiology, Oita University School of Medicine, Hasama, Yufu, Ōita-shi, Japan
}

Correspondence to: Mingqi Zheng; email: mzheng@hebmu.edu.cn

Keywords: atherosclerosis, MEK1/ERK1/2 signaling pathway, inflammatory response, macrophages, miR-223-3p

Received: February 8, $2021 \quad$ Accepted: January 11, $2022 \quad$ Published: February 24, 2022

Copyright: (C) 2022 You et al. This is an open access article distributed under the terms of the Creative Commons Attribution License (CC BY 3.0), which permits unrestricted use, distribution, and reproduction in any medium, provided the original author and source are credited.

\section{ABSTRACT}

Background: microRNAs (miRNAs) have drawn more attention to the progression of atherosclerosis (AS), due to their noticeable inflammation function in cardiovascular disease. Macrophages play a crucial role in disrupting atherosclerotic plaque, thereby we explored the involvement of miR-223-3p in the inflammatory response in macrophages.

Methods: RT-qPCR was used to analyze the miR-223-3p levels in carotid arteries and serum of AS patients. ROC curve was used to assess the diagnostic value of miR-223-3p. Movat staining was applied to evaluate the morphological differences. FISH was used to identify the expression of miR-223-3p in macrophages of atherosclerotic lesions. Bioinformatic analysis was performed. Double-immunofluorescence and western blot were performed to assess the inflammatory cytokine secretion and p-ERK1/2. C16-PAF was injected into the culture medium of the miR-223-3p mimic/NC-transfected macrophages with ox-LDL.

Results: MiR-223-3p was up-regulated in AS patients and was associated with a higher overall survival rate. MiR223-3p was co-localized with CD68+ macrophages in vulnerable atherosclerotic lesions. MiR-223-3p mimics decreased atherosclerotic lesions, macrophages numbers whereas increased SMCs numbers in the lesions. The TNF-a immune-positive areas were reduced by miR-223-3p mimics. MAP2K1 was negatively associated with miR223-3p. MiR-223-3p mimics reduced the inflammation and the MEK1/ERK1/2 signaling pathway in vivo and in vitro. C16-PAF reversed the effects of miR-223-3p mimics on inflammation and ERK1/2 signaling pathway.

Conclusions: MiR-223-3p negatively regulates inflammatory responses by the MEK1/ERK1/2 signaling pathway. Our study provides new insight into how miR-223-3p protects against atherosclerosis, representing a broader therapeutic prospect for treating atherosclerosis by miR-223-3p.

\section{INTRODUCTION}

Atherosclerosis (AS) is the cause of most cardiovascular disease types, which becomes the primary reason for morbidity and mortality worldwide $[1,2]$. Atherosclerotic lesions are characterized by the amplification of inflammatory response and accumulation of lipid droplet [2-4]. Macrophages play an essential role in the immune system and inflammatory reactions, which contribute to the development of atherosclerotic plaques with an astonishing inflammatory dysfunction [5-7].

The Raf/mitogen-activated protein kinase (MEK/MAPKK)/extracellular signal-regulated kinase $1 / 2$ (ERK 1/2) signaling pathway regulates multiple cellular processes such as proliferation, differentiation, and cell growth, and ERK phosphorylation is activated 
by MEK [8, 9]. On activation, Raf mediates phosphorylation of MEK1 and MEK2, which in turn phosphorylate ERK1 and ERK2, correspondingly [10, 11]. The activation of ERK $1 / 2$ signaling may contribute to chronic inflammation, which is involved in arteriosclerosis development $[12,13]$.

Previous studies have described an association between the modifications in microRNA (miRNA) and atherosclerosis [14-16]. MiRNAs are endogenous noncoding RNAs with a length of approximately $21 \mathrm{nt}-25 \mathrm{nt}$ (nucleotide), which control gene expression and play a critical role in vascular functions and atherosclerosis through post-transcriptional repression [14, 17]. Evidence has indicated that miRNAs target genes are related to chronic inflammation and macrophage activation [18, 19]. Several previous studies highlight that miR-223-3p is a novel prognostic marker in cardiovascular diseases $[20,21]$, and miR-223-3p can potentially affect inflammatory response and macrophage accumulation [22]. However, the role of miR-223-3p in atherosclerosis disease and its underlying molecular mechanism has not been investigated to date. The miRDIP and starBase elucidate that MEK1 was a target gene of miR-223-3p. Evidence has indicated that the MAPK signaling pathway is involved in miR-223-3p inhibition [23]. However, whether miR-223-3p involves in arteriosclerosis through down-regulating MEK1/ERK1/2 is undiscovered.

Therefore, the present study was conducted to explore the effects and regulatory mechanisms of miR-223-3p /MEK/ERK1/2 in arteriosclerosis. ApoE-/- mice were fed with a high-fat diet (HFD) to induce AS models, macrophages treated with ox-LDL were served as cell AS models.

\section{RESULTS}

\section{Enhanced MiR-223-3p levels in carotid arteries and serum from patients with AS and co-localization with macrophages in vulnerable atherosclerotic lesion}

To evaluate the expression level of miR-223-3p as potential biomarkers of AS, the information of the patients were shown in Table 1. The qPCR results revealed that the expression level of miR-223-3p was upregulated in both serum and carotid arteries samples of AS patients compared with controls. (Figure 1A, 1B, $\mathrm{P}<0.05$ ). Furthermore, we performed the receiver operating characteristic (ROC) curve to assess the diagnostic value of miR-223-3p. We found that the expression level of miR-223-3p is associated with a higher overall survival rate, suggesting that the miR-223$3 p$ could be an excellent diagnostic marker for AS (Figure 1C). And the medical imaging were shown in Figure 1D. Consequently, we carried out a histological analysis on the carotid artery sections to evaluate the morphological differences using Movat staining. Representative staining results for vulnerable plaque and stable plaque were exhibited in Figure 1E.a, b, and we found the vulnerable plaque was associated with increased medial thickness and luminal diameter, and the messages about the patients were shown in Table 1. Additionally, we also found reduced miR-223-3p expression levels in the vulnerable plaque compared with stable plaque (Figure 1E.c). In addition, we carried out a Fluorescent in situ Hybridization (FISH) assay to identify the miR-223-3p in macrophages of the intima and vulnerable atherosclerotic lesions. We found miR-223-3p co-localization with $\mathrm{CD} 68+$ macrophages in vulnerable atherosclerotic lesions of AS patients (Figure 1F).

\section{Prevention of AS progression and inflammation by miR-223-3p mimics}

To functionally investigate the role of miR-223-3p in vulnerable atherosclerotic lesions, we applied the wellestablished mice atherosclerosis HFDs, in which atherosclerotic lesions were induced in apolipoprotein-E deficient $\left(\mathrm{ApoE}^{-/}\right)$mice by a high-fat diet. To further elucidate the in vivo effects of miR-223-3p in AS, ApoE ${ }^{-/}$ mice were assigned to high-fat diet (HFD) + miR-223-3p negative control (NC), and HFD + miR-223-3p mimics. Mice carotid roots and myometrial tissues were obtained and stained with Movat staining. Representative staining results were shown in Figure 2A, atherosclerotic lesions were shown in both groups, and an obvious decrease in the extent of atherosclerotic lesions in the miR-223-3p mimics. Moreover, the plaque area staining positive for macrophages $(\mathrm{CD} 68+)$ in miR-223-3p mimics decreased compared with the NC group, whereas the SMA staining level was increased in miR-223-3p mimics (Figure 2B, $2 \mathrm{C}$ ), suggesting that miR-223-3p mimics could reduce macrophages accumulation and promote smooth muscle cells composition. ELISA results revealed that the TNF-a immunopositive plaque area also reduced in miR-223-3p mimics compared with the NC group (Figure 2D). The quantitative analysis was consistent with the above findings obtained in our in vitro experiments. $(\mathrm{P}<0.05$, miR-223-3p mimics vs. miR-223-3p NC). In brief, these in vivo experimental data implied that miR-223-3p mimics could prevent AS from developing.

\section{Targeting relationship between miR-223-3p and MEK/ERK}

Given that miRNAs exert their function by reducing their downstream expression of target genes [24]. The variety of functions of miR-223 has been linked to the suppression of many different target genes in inflammatory pathologies $[25,26]$. Therefore, miR2233 p may affect cell differentiation and activation by 
Table 1. Clinicopathological characteristics of atherosclerotic patients.

\begin{tabular}{lcc}
\hline Characteristics & Stable group & Vulnerable group \\
\hline Age & $64.25 \pm 3.038 \mathrm{~N}=4$ & $69.50 \pm 2.630 \mathrm{~N}=4$ \\
Gender & Male:3/Femal:1 & Male:3/Femal: 1 \\
Have hyperlipidemia & $\mathrm{N}=4$ & $\mathrm{~N}=4$ \\
Have diabetes & $\mathrm{N}=1$ & $\mathrm{~N}=2$ \\
Have hypertension & $\mathrm{N}=2$ & $\mathrm{~N}=3$ \\
Maximum diameter under ultrasonic $(\mathrm{mm})$ & $1.098 \pm 0.093 \mathrm{~N}=4$ & $3.283 ? 0.2463 \mathrm{~N}=4 * *$ \\
\hline
\end{tabular}

suppressing its target genes, which have been protective against inflammatory response. We obtained three potential genes (PLAGL2, FLOT2, and MAP2K1) of miR-223-3p by combining the results from the online miRNA target prediction databases (Figure 3A). To confirm whether the three potential genes were the downstream target genes for miR-223-3p, we examine the expression of the three potential genes in macrophages transfected with miR-223-3p mimics. The qPCR results revealed that MAP2K1(MEK1) expression was noticeably decreased in macrophages transfected with miR-223-3p mimics (Figure 3B). Furthermore, miRDIP and starBase databases were applied, and we found miR-223-3p and MAP2K1 had targeted binding regions (Figure 3C). There results suggested that MAP2K1 was a direct target of miR-223$3 p$. Upon activation, Raf mediates phosphorylation of MEK1 and MEK2, which in turn phosphorylate ERK1 and ERK2 [27]. Thus, we hypothesize that the regulation of miR-223-3p may be through the MEK/ERK signaling pathway in atherosclerosis. To assess the inflammatory response signaling pathway in AS, we downloaded the GSE34822 dataset from the GEO database, comprised of atherosclerosis samples. As shown in Figure 3D, 158 up-regulated differentially expressed genes (DEGs) and 206 down-regulated DGEs were obtained using the criteria of $\operatorname{Padj}<0.01,|\log 2 \mathrm{FC}|$ $>1.2$, hierarchical clustering analysis exhibited the distinction on differentially expressed genes in the heat map. We then performed pathway analysis by David and established Go enriched up-regulated pathways (Figure 3E), including inflammatory response, metabolic process, lipid transport, and intracellular protein transport. We also found down-regulated pathways, including anion transmembrane transport, glycerol transport, and ADP biosynthetic process. (Figure 3F) Correspondingly, relevant partial results for KEGG pathways were exhibited in Figure 3G. Furthermore, the gene set enrichment analysis (GSEA) revealed that the innate immune pathway regulation was functionally enriched in AS (Figure $3 \mathrm{H}$, 3I). Thus, we pick up miR-223-3p, MEK/ERK1/2, and the inflammatory response pathway for further investigation.

\section{Regulation of inflammatory cytokine secretion and p-ERK1/2 by miR-223-3p}

Double-immunofluorescence was performed on carotid tissues from 223-3p mimics and the NC mice group. As shown in Figure 4A, the double-immunofluorescence exhibited significant co-localization of the macrophage marker CD68+ and TNF- $\alpha$, suggesting that the macrophages express TNF- $\alpha$ in miR-223-3p mimics (Figure 4A). To further investigate the possible role of miR-223-3p in the inflammatory response of AS, protein expression changes in mice carotid tissues were determined by quantitative analysis and western blots, respectively. We found significantly decreased expression levels of inflammatory cytokine (IL-6 and TNF-a) in carotid tissues from the miR-223-3p mimics group in western blots (Figure 4B). Additionally, we also found significantly reduced p-ERK $1 / 2$ expression level in miR-223-3p mimics. (Figure 4B). The quantitative analysis of relative protein expression confirmed the associated decrease in the protein levels of IL-6, TNF-a, and ERK1/2 in carotid tissues of miR223-3p mimics as compared with the NC group (Figure $4 \mathrm{~B}, \mathrm{P}<0.05$ ), suggesting that miR-223-3p mimics could repress inflammatory response and phosphorylation ERK1/2 in AS.

\section{MiR-223-3p mimics inhibits the inflammatory response via the ERK1/2 pathway in vitro}

The macrophages might initiate and exacerbate inflammation with ox-LDL stimulation and prompt the destabilization of the atherosclerotic plaques [28]. To investigate the biological importance of ERK1/2 as a target of miR-223-3p, miR-223-3p mimic/NCtransfected macrophages were stimulated with ox-LDL and saline to detect the ERK1/2, IL6, TNF-a expression in in vitro. Using western blots, we found that the expression levels of p-ERK1/2, IL6, and TNF-a were significantly suppressed in miR-223-3p mimictransfected macrophages with ox-LDL stimulation as compared with miR-223-3p NC-transfected macrophages with ox-LDL (Figure 5A). The result of in vitro implied that miR-223-3p mimic could repress 
A

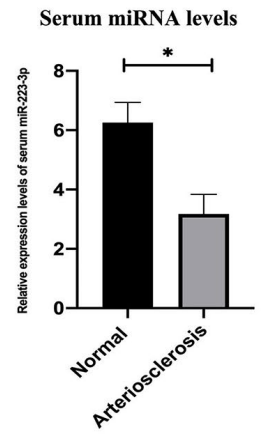

D
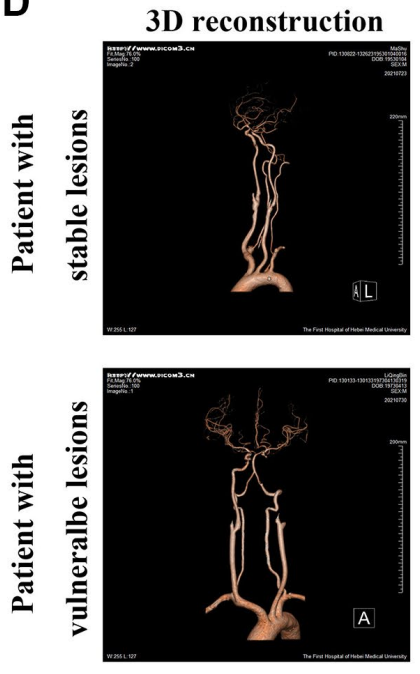

E
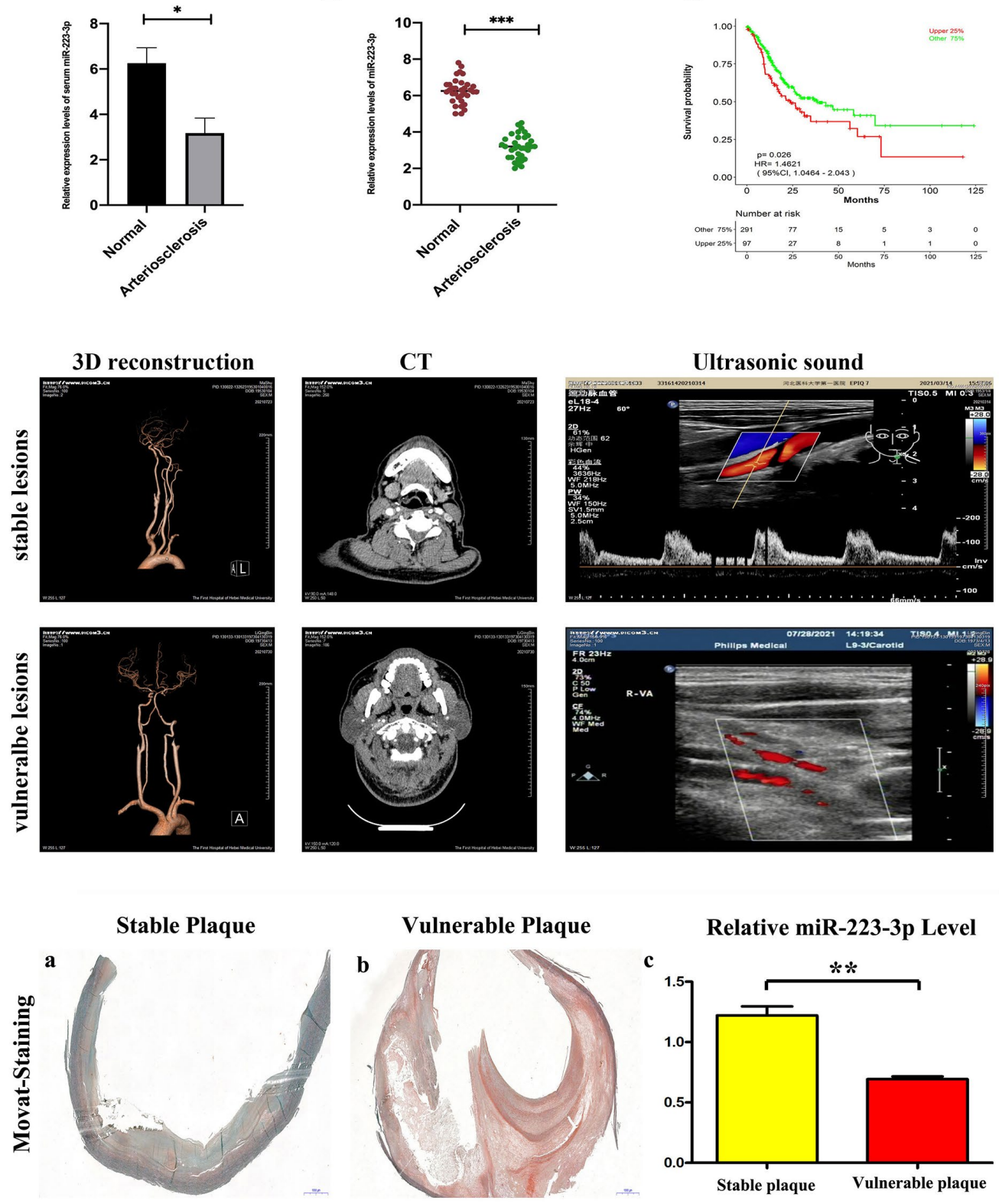

C

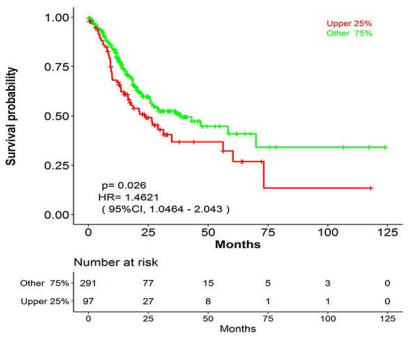

Ultrasonic sound

Relative miR-223-3p Level

c

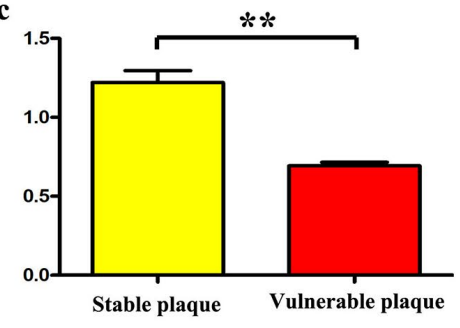

$\mathbf{F}$

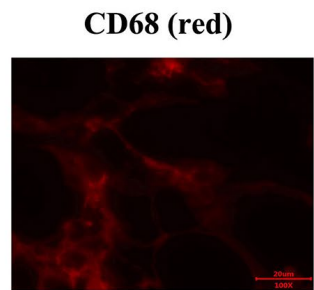

miR-223-3p (green)

DAPI (blue)

Merge
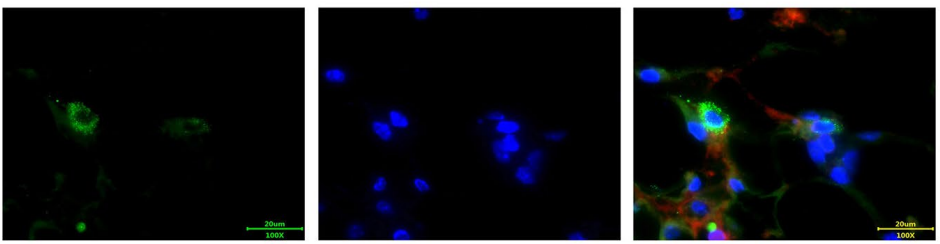

Figure 1. Enhanced miR-223-3p levels in carotid arteries and serum from patients with AS. (A) The levels of miR-223-3p were higher in AS arteries as compared with normal arteries determined by $q P C R$. ( $\left.{ }^{*} P<0.05\right)$. (B) The levels of miR-223-3p were higher in the serum of AS patients compared with normal controls determined by qPCR. ( $\left.{ }^{*} P<0.05\right)$. (C) The expression level of miR-223-3p has been associated with a higher overall survival rate by ROC curve. (D) The three-dimensional vascular remodeling, CT, ultrasonic sound pictures from patient with stable or vulnerable lesions were shown. (E) Histological analysis on the aortic sections evaluates the morphological differences by Movat staining. Representative staining results for vulnerable plaque and stable plaque. Decreased miR-223-3p in the vulnerable plaque as compared with stable plaque $\left({ }^{*} \mathrm{P}<0.05\right)$. (F) Fluorescent In Situ Hybridization assay revealed miR-223-3p (green) co-localization with CD68+ (red) macrophages in vulnerable atherosclerotic lesions of AS patients. DAPI (blue) for nuclei staining. 
the inflammatory response in AS. To further determine whether miR-223-3p mimic mediated inflammatory effect in ox-LDL stimulated macrophages was via ERK1/2 signaling pathway, C16-PAF, an ERK1/2 agonist was injected into the culture medium of the miR-223-3p mimic/NC-transfected macrophages with ox-LDL stimulation. Interestingly, we found that the decreased expression levels of p-ERK1/2, IL6, and TNF-a in miR-223-3p mimic-transfected macrophages with ox-LDL were markedly reversed by C16-PAF, suggesting that miR-223-3p mimic mediated inflammatory effect in ox-LDL stimulated macrophages was via ERK1/2 signaling pathway (Figure 5A). As shown in Figure 5B, the quantitative analysis of relative protein expression was consistent with our western blot analysis data $(\mathrm{P}<0.05$, Figure $5 \mathrm{~B})$. Therefore, these data suggested the essential role for ERK $1 / 2$ as a mediator of the biological effects of miR-223-3p-mediated inflammatory effect in AS.

\section{DISCUSSION}

AS is an inflammatory diseases, the macrophages play a significant roles in the formation and progression of atherosclerosis, therefore the factors regulated the inflammatory factors and associated modulatory pathway proteins secreted by lesional macrophages, deserved of research [29, 30]. Previous studies demonstrated the miRNAs involved in atherosclerotic progression and also played crucial effects in modulating lesional inflammatory responses [31-33]. In the current study, Firstly, bioinformatics analysis were used for screening out the miRNA-223-3p involved in the progression of AS, the expression level of miR-223$3 p$ was up-regulated in both carotid arteries and serum of AS patients, the miR-223-3p is associated with a higher overall survival rate, suggesting that the miR-223-3p could be an excellent diagnostic marker for AS, data shown in Figure 1A-1C. And further the
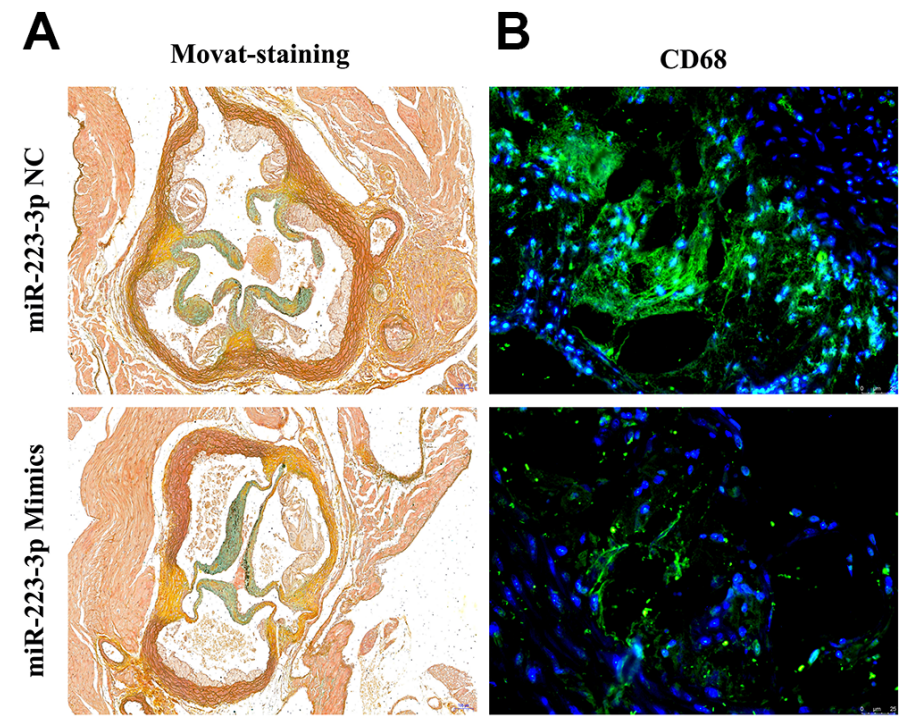

Atherosclerotic lesional areas

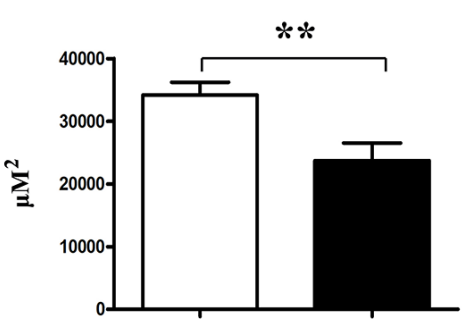

Relative fluorescence intensity of CD68

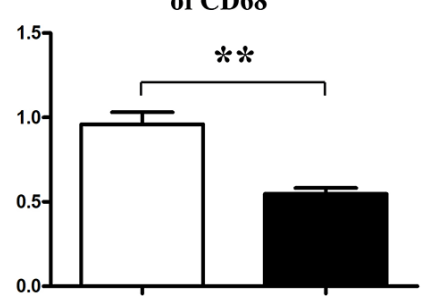

C
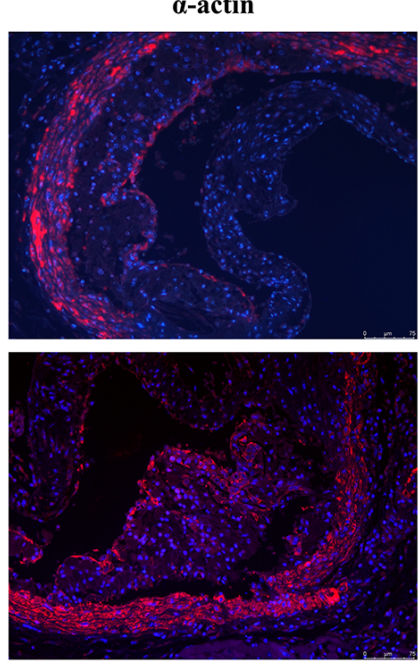

Relative fluorescence intensity of $\alpha$-actin

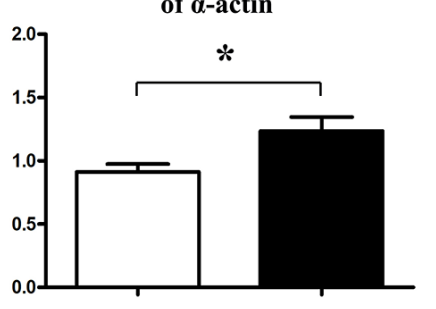

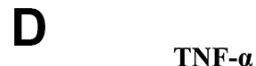
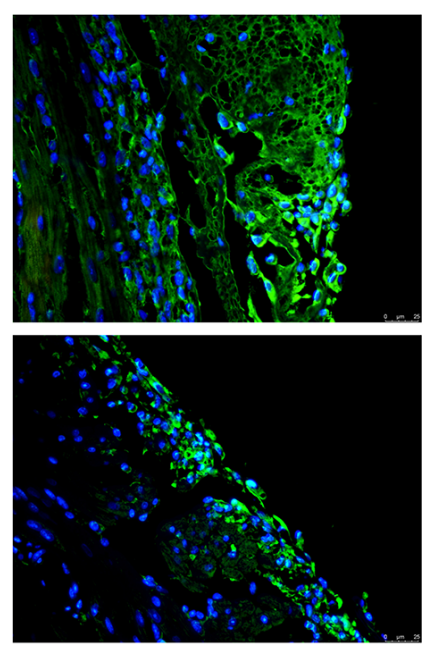

$\square$ miR-223-3p NC miR-223-3p Mimics

Relative fluorescence intensity of TNF-a

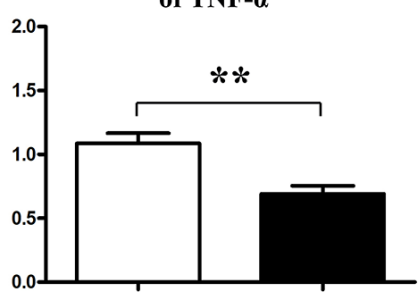

Figure 2. Prevention of AS progression by miR-223-3p mimics. (A) Representative Movat staining of the histopathology of mice's carotid roots and a pronounced decrease in the extent of atherosclerotic lesions in the carotid roots tissues from miR-223-3p mimics. $\left({ }^{*} \mathrm{P}<0.05\right)$. (B) The plaque area staining positive for macrophages (CD68) in the miR-223-3p mimics group and NC group (*P<0.05). (C) The SMA staining in mice's aortic roots in the miR-223-3p mimics group and NT group $\left({ }^{*} P<0.05\right)$. (D) TNF- $\alpha$ staining in mice's aortic roots in the miR-223-3p mimics group and NT group $\left({ }^{*} \mathrm{P}<0.05\right)$. 
Q-PCR were used for testing the mRNA levels of miR223-3p in human species of stable and vulnerable atherosclerotic plaques, shown in Figure 1E. Besides, Fluorescent In Situ Hybridization (FISH) assay revealed miR-223-3p co-localization with CD68+ macrophages in vulnerable atherosclerotic lesions of AS patients, as showed in Figure 1F, thereby the macrophages were elected for target cellular type in our study.

The disease based on atherosclerosis, such as myocardial infarction, apoplexy, cerebral infarction, obliterans of the Lower Extremities and et al., attracted from youth to middle aged and old aged people [34, 35]. The vulnerable/ instable plaques mainly appeared in old persons [36] and in this study, the atherosclerotic lesions from patients of the first affiliated hospital of Hebei medical university were obtained by carotid endarterectomy (CEA). Consequently, we carried out a histological analysis of aortic sections from AS patients to evaluate the morphological differences, representative Movat staining results for vulnerable plaque and stable plaque were exhibited in Figure 1D, and we found decreased miR-223-3p expression level in the vulnerable plaque compared with stable plaque,
A

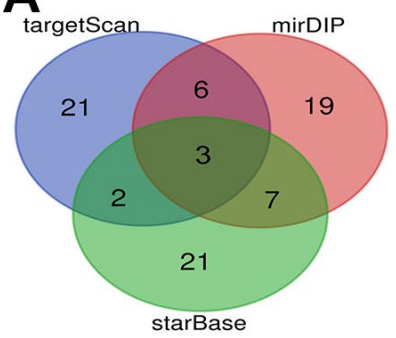

D

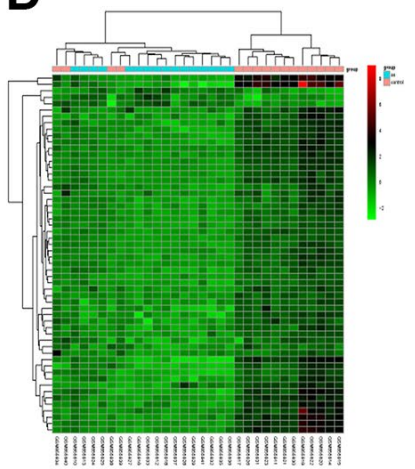

B

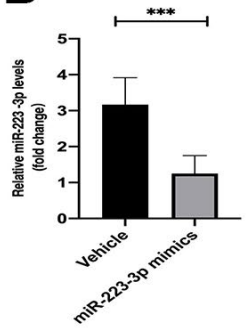

C

\author{
MAPK1: 5' uguacccucgcauGACUGUUa 3' \\ |||||| \\ hsa-miR-223-3p: $3^{\prime}$ ccggcacugaccuCUGACAAu $5^{\prime}$
}

E

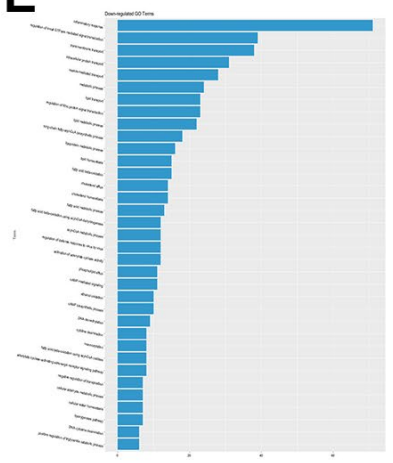

$\mathbf{F}$

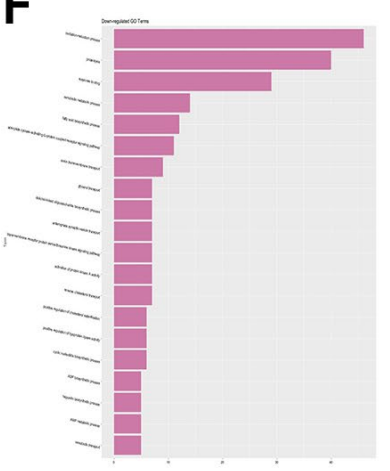

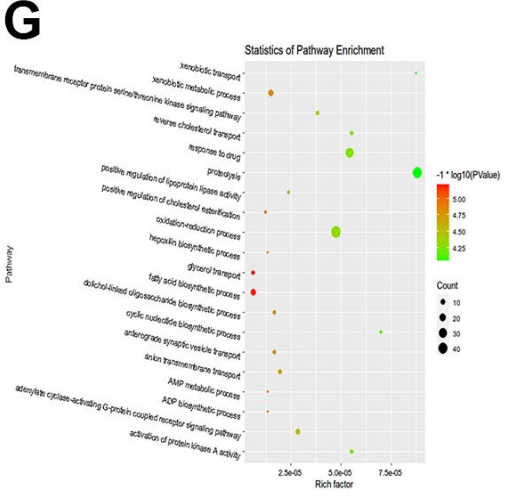

\section{H}
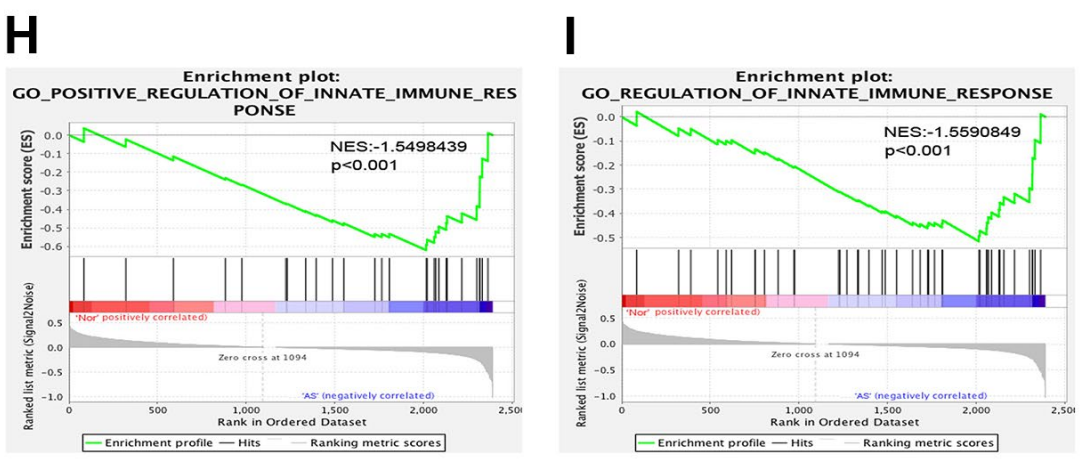

Figure 3. Targeting relationship between miR-223-3p and MEK/ERK. (A) The miRNA target prediction databases exposed three potential genes (PLAGL2, FLOT2, and MAP2K1) of miR-223-3p. (B) The qPCR revealed that MAP2K1(MEK1) expression was noticeably decreased in macrophages transfected with miR-223-3p mimics. (C) miRDIP and starBase databases showed that miR-223-3p and MAP2K1 had targeted binding regions. (D) The hierarchical clustering analysis exhibited the distinction on differentially expressed genes in the heat map. (E) Go enriched up-regulated pathways. (F) Go enriched down-regulated pathways. (G) The relevant partial results for KEGG pathways. (F) The gene set enrichment analysis (GSEA) revealed that the innate immune pathway regulation was functionally enriched in AS. (H, I) GSEA revealed that the innate immune pathway regulation was functionally enriched in AS. 
indicating that miR-223-3p has the value to be made further research on. The detailed mechanisms of miR223-3p were further verified in animal models, we applied the well-established atherosclerotic mouse models, apolipoprotein-E deficient (ApoE-/-) mice, in which AS lesions were induced by fed with high fat diet. $\mathrm{ApoE}^{-/-}$mice were treated with miR-223-3p negative control and mimic and pathological analysis results showed that the increase of miR-223-3p decreased lesional areas following with macrophage decrease, TNF- $\alpha$ secretion and smooth muscle cells increase. Lesional macrophages is the major cellular resource responding for inflammatory factors secretion, TNF- $\alpha$, IL-6, IFN- $\gamma$, et al., resulting in accumulating
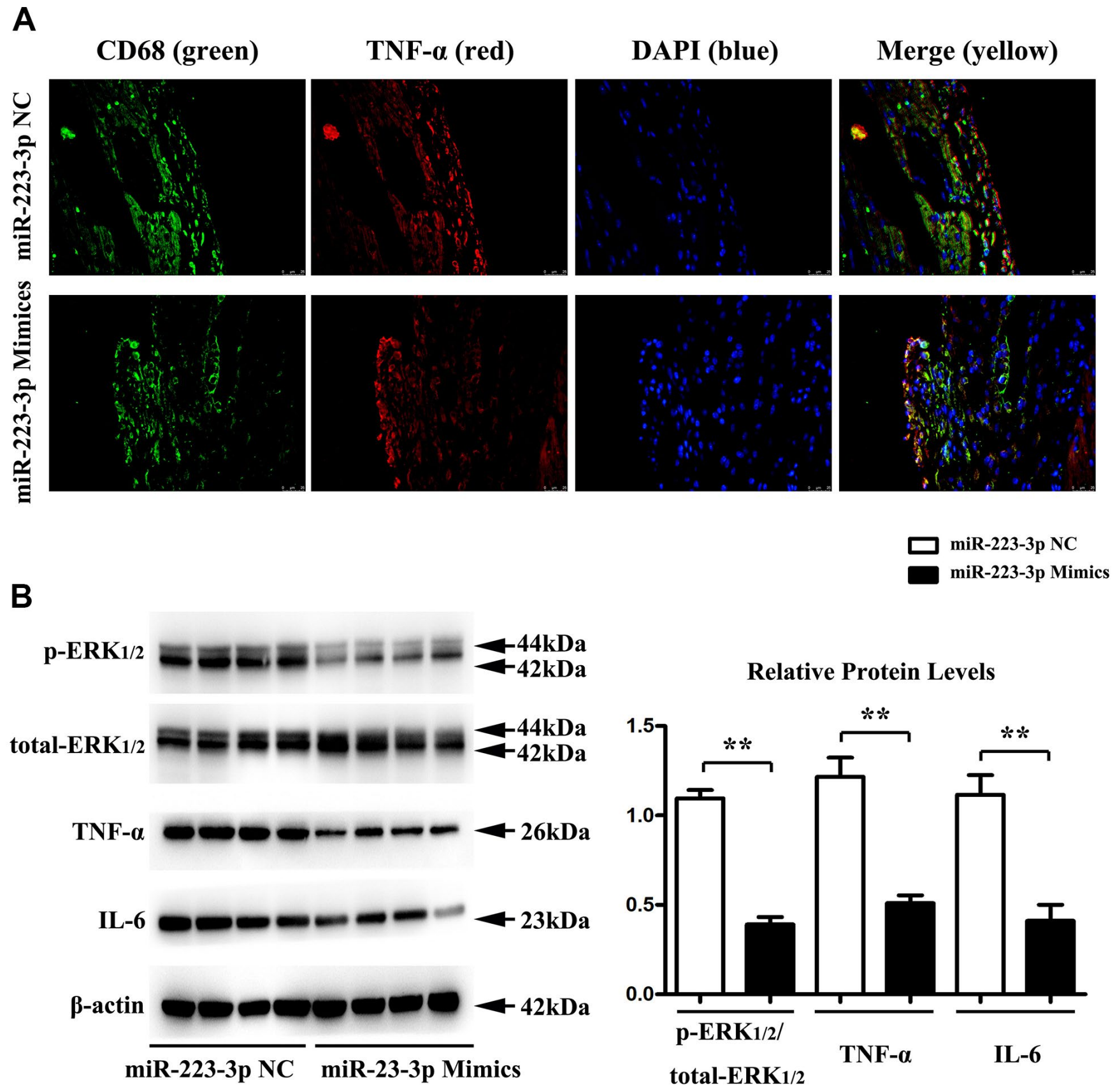

Figure 4. Regulation of inflammatory cytokine secretion and p-ERK1/2 by miR-223-3p. (A) Double-immunofluorescence revealed significant co-localization of the macrophage marker CD68+ and TNF- $\alpha$ in carotid tissues from 223-3p mimics and. (B) The western blots analysis revealed significantly decreased expression levels of IL-6, TNF-a, and p-ERK1/2 in carotid tissues from the miR-223-3p mimics group. The quantitative analysis of relative protein expression exposed the associated decrease in the protein levels of IL-6, TNF-a, and ERK1/2 in carotid tissues of miR-223-3p mimics ( $\left.{ }^{*} \mathrm{P}<0.05\right)$. 
A
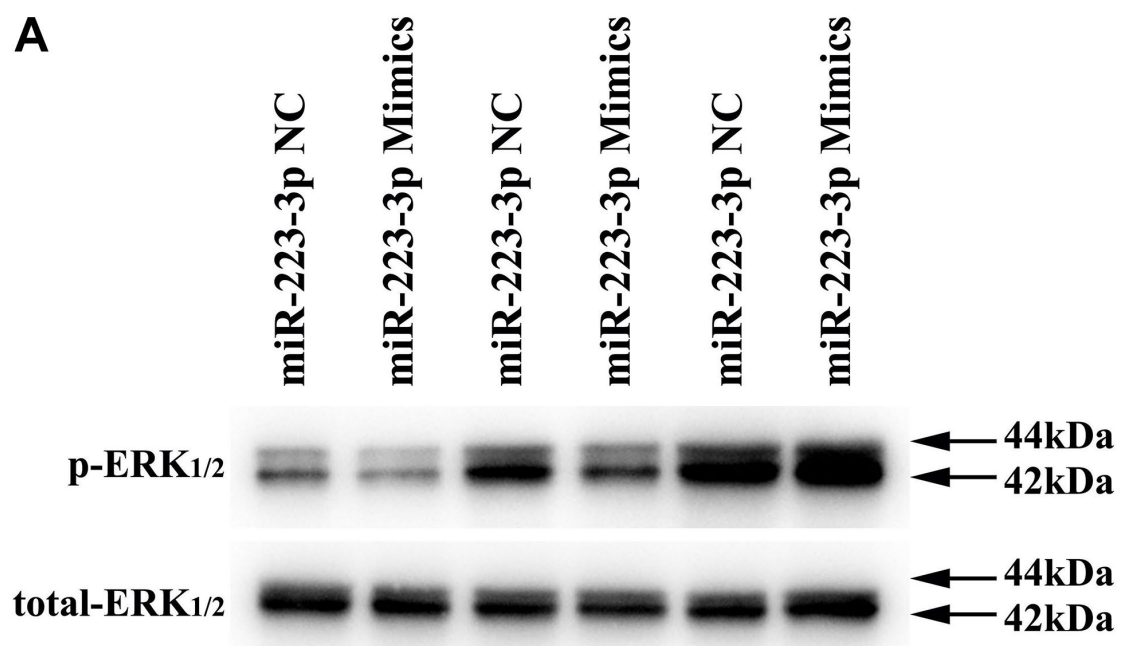

TNF-a $-\longrightarrow 26 \mathrm{kDa}$
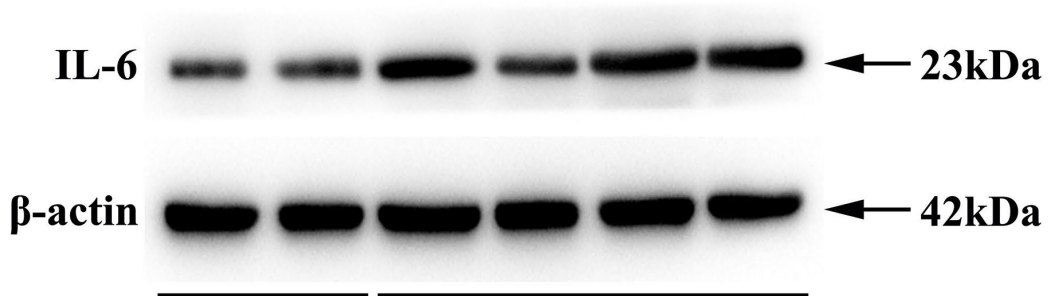

$(-) \quad 75 \mu \mathrm{g} / \mathrm{ml} \mathrm{ox}-\mathrm{LDL}$

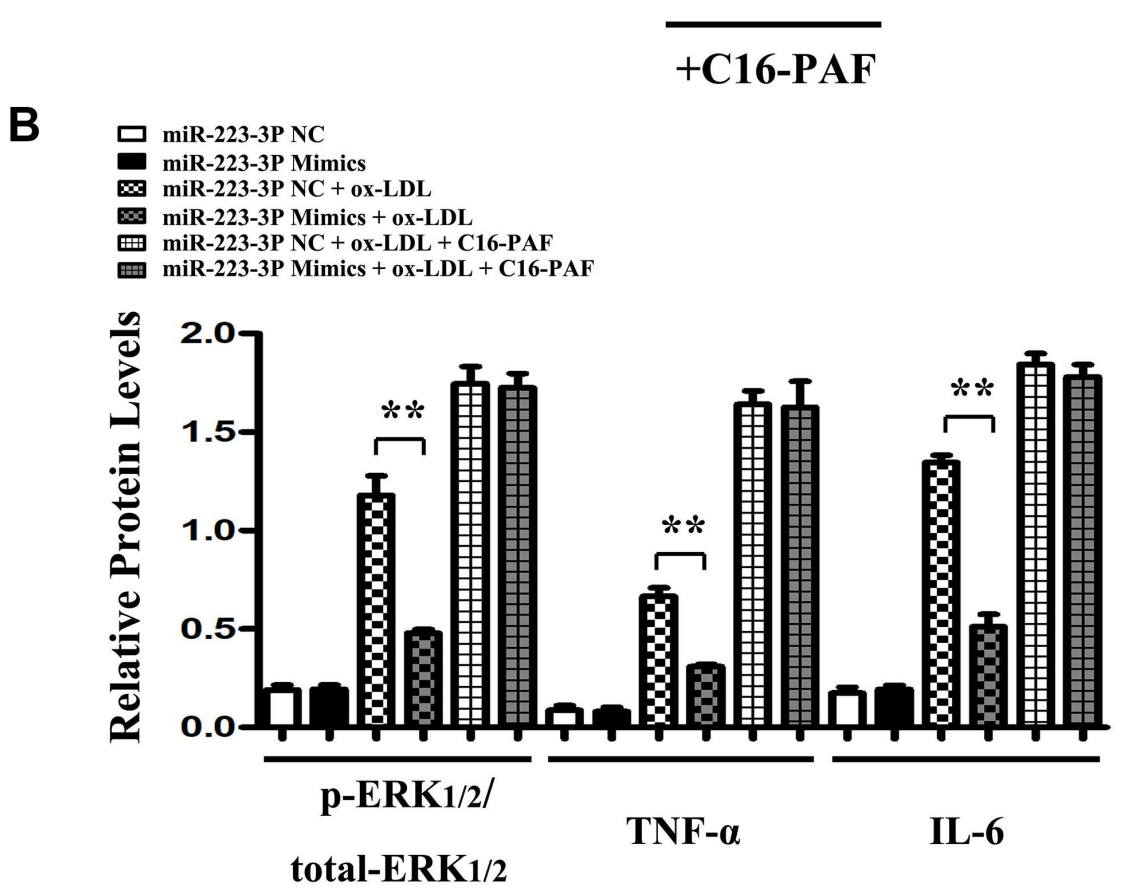

Figure 5. MiR-223-3p mimics inhibits the inflammatory response was via the ERK1/2 pathway in vitro. (A) The western blots analysis revealed that $p$-ERK1/2, IL6, and TNF-a were significantly suppressed in miR-223-3p mimic-transfected macrophages with ox-LDL stimulation, and the decreased expression levels of p-ERK1/2, IL6, and TNF-a were markedly enhanced in miR-223-3p mimic-transfected macrophages with ox-LDL by C16-PAF. (B) The quantitative analysis revealed that the relative protein levels of p-ERK1/2, IL6, and TNF-a were decreased in miR-223-3p mimic-transfected macrophages with ox-LDL stimulation, and the decreased relative protein levels of p-ERK1/2, IL6, and TNF-a were markedly enhanced in miR-223-3p mimic-transfected macrophages with ox-LDL by C16-PAF. ( $\left.{ }^{*}<0.05\right)$. 
circulating monocytes and macrophages into AS and promoting outbreak of inflammatory response [37-39], thus forming a vicious cycle; and the lesional SMC is the major cell secreting collagen, extracellular matrix components, the principal contents of fibrotic caps, protected the vulnerable plaques from rupture, playing protective roles [40, 41]. Our vivo experimental data illustrated that miR-223-3p mimics-mediated inhibition of inflammatory responses is one of the mechanisms involved in the development of AS.

MiRNAs exert their function by reducing their downstream expression of target genes. The variety of functions of miR-223 has been related to the suppression of many different target genes in inflammatory pathologies [25]. Therefore, miR-223-3p may affect cell differentiation and activation by suppressing its target genes, which have been protective against inflammatory response. We obtained the potential gene MAP2K1 of miR-223-3p by combining the results from the online miRNA target prediction databases. The qPCR results revealed that MAP2K1(MEK1) expression was noticeably decreased in macrophages transfected with miR-223-3p mimics. Furthermore, miRDIP and starBase databases were applied, miR-223-3p and MAP2K1 had targeted binding regions. There results suggested that MAP2K1 was a direct target of miR-223-3p. Upon activation, Raf mediates phosphorylation of MEK1 and MEK2, which in turn phosphorylate ERK1 and ERK2 [27]. Thus, we hypothesize that the regulation of miR-223-3p may be through the MEK/ERK signaling pathway in atherosclerosis. To assess the inflammatory response signaling pathway in AS, we downloaded the GSE34822 dataset from the GEO database, comprised of atherosclerosis samples. The hierarchical clustering analysis exhibited the distinction on differentially expressed genes in the heat map according to the GSE34822 dataset. We then performed pathway analysis by David and established Go enriched upregulated and down-regulated pathways and relevant partial results for KEGG pathways. The gene set enrichment analysis (GSEA) revealed that the innate immune pathway regulation was functionally enriched in AS. Thus, we pick up miR-223-3p, MEK/ERK1/2, and the inflammatory response pathway for further investigation.

Double-immunofluorescence exhibited significant colocalization of the macrophage marker $\mathrm{CD} 68+$ and inflammatory factor TNF- $\alpha$, indicating the TNF- $\alpha$ secreted by macrophage in AS lesions. To further investigate the possible role of miR-223-3p in the inflammatory response of AS, protein expression changes in mice carotid tissues were determined by quantitative analysis and western blots. Respectively, we found significantly decreased expression levels of inflammatory cytokine (IL-6 and TNF-a) in carotid tissues from the miR-223-3p mimics group compared with the $\mathrm{NC}$ group. Evidence has indicated that the MAPK1 signaling pathway is involved in miR-223-3p inhibition by combining with 3'-UTR of MAPK1 for further degradation [23, 42]. As the MAPK signals especially the MAPK1/ERK2 is the major modulatory signal proteins for inflammatory factors synthesis and sections [43, 44]. Using quantitative analysis and western blots, we found a significantly decreased $\mathrm{p}$ ERK1/2 expression level in miR-223-3p mimics compared with the NC group $(\mathrm{P}<0.05)$, suggesting that miR-223-3p mimics could repress inflammatory response and phosphorylation ERK1/2 in AS, these results were consistent with our bioinformatics analysis.

The macrophages might initiate and exacerbate inflammation with ox-LDL stimulation and prompt the destabilization of the atherosclerotic plaques [28]. Using western blots analysis, we found that the expression levels of p-ERK1/2, IL6, and TNF- $\alpha$ were significantly suppressed in miR-223-3p mimictransfected macrophages with ox-LDL stimulation as compared with miR-223-3p NC. C16-PAF, an ERK1/2 agonist, was injected into the culture medium of the miR-223-3p mimic/NC-transfected macrophages with ox-LDL stimulation to investigate whether miR-223-3p mimic mediated inflammatory effect was via ERK1/2 signaling pathway. Interestingly, we found that the decreased expression levels of p-ERK1/2, IL6, and TNF-a were markedly enhanced in miR-223-3p mimictransfected macrophages with ox-LDL by C16-PAF, suggesting that miR-223-3p mimic mediated inflammatory effect was via ERK1/2 signaling pathway. These results implied that the MEK/ERK1/2 signaling pathway was necessary to facilitate the development of miR-223-3p in AS. Our in vitro experiment demonstrated that miR-223-3p could play an antiatherosclerosis role by modulating ERK activity and inflammatory factor secretion.

In conclusion, our study elucidates that miR-223-3p prevents the development of AS and reduced inflammatory response through down-regulation of MEK1/ERK1/2, which suggests that miR-223-3p /MEK1/ERK1/2 can be potentially used as an attractive therapeutic for AS.

\section{MATERIALS AND METHODS}

\section{Patients and specimens}

8 patients with stable plaques $n=4$, and vulnerable plaques $\mathrm{n}=4$ underwent diagnostic carotid CT and ultrasonic testing to intent to revascularize the culprit arteries shown 
in Figure 1B. All patients presented with atheromatous plaques of the extracranial artery but without thrombus, the information of risk factors for atherosclerosis such as diabetes and hypertension were also collected. The detailed patient information were collected.

\section{Establishment of mice AS models}

The ApoE ${ }^{-/}$mice (C57B1/6 background) were obtained from Beijing HFK Biotechnology Co., Ltd (Beijing, China). The animal use protocol for this study has been reviewed and approved by the Laboratory Animal Ethical Committee, Hebei Medical University first Affiliated Hospital. ApoE ${ }^{-/-}$mice established adeno-associated virus serotype-9 (AAV9) mediated miR-223-3p overexpression via the tail vein. The groups were set as follows: high-fat diet (HFD) + miR-223-3p negative control (NC), and HFD + miR$223-3 p$ mimics groups via tail vein injection. The mice were fed a high-fat diet (HFD) to induce early atherosclerotic lesions. After providing a high-fat diet for two months and transfection with AAV9, the mice were sacrificed.

\section{Enzyme-linked immunosorbent assay}

After injection with pentobarbital sodium, blood samples were gathered from the carotid artery of AS patients and controls. The serum contents were treated with regional citrate anticoagulation (RCA) and centrifuged at $2500 \mathrm{~g}$ for $20 \mathrm{~min}$ at four ${ }^{\circ} \mathrm{C}$. Blood cells were recovered by centrifugation at $700 \times \mathrm{g}$ for $20 \mathrm{~min}$ at $4^{\circ}$ C. The ELISA kit (Cat. No. 210-A-050, R\&D Systems, Minneapolis, MN) was conducted to identify the plasma and macrophages expressed according to the kit instructions. The serum levels of inflammatory cytokines interleukin-6 (IL-6) (eBioscience) and tumor necrosis factor- $\alpha$ (TNF- $\alpha)$ (eBioscience) were examined using ELISA kits (RapidBio, CA, USA).

\section{Movat-staining}

After thoroughgoing washing with water, the aortic tissues were rinsed by $60 \%$ isopropanol for $5 \mathrm{~min}$ and stained with the Movat-staining dyeing solution for 2 $\mathrm{h}$, then differentiated and rinsed by $60 \%$ isopropanol for $6 \sim 7$ times. Finally, the images were captured under an optical microscope $(\times 400$, Olympus Optical Co., Ltd.).

\section{Macrophage culture and transfection}

Mouse macrophage-like cell line RAW 264.7 was obtained from Peking Union Medical College. After adding Dulbecco's modified eagle's medium (DMEM, General Electric Company, Utah, USA) with $10 \%$ fetal bovine serum (FBS) (Gibco Company, Grand Island, NY, USA), the cells were cultured at $37^{\circ} \mathrm{C}$ with $5 \% \mathrm{CO} 2$. Forty-eight hours later, the cells were treated with $0.25 \%$ trypsin (Biotime Biotechnology) and re-cultured in a $75 \mathrm{~mL}$ flask. The cells were seeded in 6-well plates at $2.5 \times 105$ cells per well and cultured in a medium before transfection. According to the manufacturer's instructions, cells were then transfected using Lipofectamine 2000 (Santa Cruz Inc., Santa Cruz, CA, USA). The miRNA and Lipofectamine 2000 reagent were individually diluted to $20 \%$ in serum-free DMEM and incubated for 10 mins. Then cultured macrophages were transfected with miR-223-3P mimics, miR-223-3P mimics negative control (NC). The primary culture medium was detached three hours after transfection, and the cells were cultured for an additional 24 hours in the transfection complex. Twenty-four hours after transfection, the culture medium was removed, and the cells were re-cultured for an extra 48 hours. Consecutively diluted ox-LDL at 50,100, and 200 $\mathrm{mg} / \mathrm{L}$ were used to treat the cells for $12 \mathrm{~h}, 24 \mathrm{~h}$, and 48 h. qPCR was conducted on a Step One Plus system and Chromo4 using specific primers (Applied Biosystems, Foster City, CA). Values of miR-223-3P were normalized (cultured macrophages) and presented as $2-\Delta \Delta \mathrm{CT}$ equation through boxplot with smallest and largest whiskers.

\section{Fluorescent In Situ Hybridization (FISH) assay}

The FISH assay was applied to detect the location and expression of miR-223-3P in aortic tissues. The aortic tissues were fixed with $4 \%$ paraformaldehyde at room temperature and embedded in paraffin $(4-\mu \mathrm{m}$ thickness). Subsequently, the tissues were deparaffinized by mineral oil. They rehydrated in graded dilutions of ethanol $(100 \%, 100 \%, 90 \%, 80 \%$, $70 \%, 50 \%$ ), digested with $16 \mu \mathrm{g}$ of proteinase $\mathrm{K}$ (Thermo Fisher Scientific, San Jose, CA, USA) at $37^{\circ} \mathrm{C}$ for $15 \mathrm{~min}$, rinsed in $0.2 \%$ glycine in $\mathrm{PBS}$ (Sigma-Aldrich) for $10 \mathrm{~min}$, fixed with $4 \%$ paraformaldehyde phosphate-buffered saline (PBS) at room temperature for $10 \mathrm{~min}$. Then, paraffin slides were hybridized with fluorescent (Cy3-labeled) oligonucleotide miR-223-3P probes (5'-UGUCA GUUUGUCAA AUACCCC-3') at $37^{\circ} \mathrm{C}$ overnight. After probe hybridized, tissues were incubated in extraction buffer $(20 \mathrm{mM}$ Tris-HCl, $\mathrm{pH} 7.5,2 \mathrm{mM}$ EDTA, $0.25 \%$ SDS, $0.225 \mathrm{M} \mathrm{NaCl}$ ), at $37^{\circ} \mathrm{C}$ for 20 min. Tissues were stained with CD68+ with purified ab81289 (Abcam Inc., Cambridge, MA) at 1:200. The conventional heat-mediated antigen retrieval was conducted with Tris-EDTA buffer $(\mathrm{pH}$ 7.6, Abcam Inc., Cambridge, MA). Goat Anti-Rabbit IgG H\&L (HRP) was used as the secondary antibody at 
1/5000 dilution. 4,6-diamidino-2-phenylindole (DAPI) staining was used for cell nuclear counterstain with laser scanning confocal microscopy (Olympus, Japan). A light microscope carried out the qualitative analysis ((Nikon Corporation, Tokyo, Japan). Images of the sections were captured by fluorescence microscope (magnification, $\times 200$; BX50, Olympus) and analyzed using Image-Pro Plus 6.0 software (Media Cybernetics, USA).

\section{Western blot analysis}

The protein extracts from aortic tissues and cells were denatured. The protein samples were separated by SDSPAGE $(10 \%$ gel) and transferred to PVDF membranes (Millipore, MA, USA). After washing with Trisbuffered saline with Tween- 20 (TBST), the protein samples were incubated with primary and secondary antibodies (Abcam) for 1h. Band intensities were detected by an Odyssey infrared imaging system (Licor, Lincoln, NE, USA).

\section{RNA isolation and real-time PCR}

Total RNA was isolated from macrophages and aortas using a Trizol reagent (Life Technologies, Inc, Burlington, ON, Canada) and purified by the RNA Easy kit (Qiagen Inc., Valencia, CA). qPCR fluorophore SYBR-Green was purchased from Beijing Solarbio Science and Technology Co., Ltd. Reverse transcriptionquantitative PCR (qRT-PCR) of the target mRNA was carried out using primers, The mice primers: miR-223-3p' F 5'-GTG CAG GG TCC GAG GT-3';R 5'-CGG GCT GTC AGT TTG TCA-3'; U6' F 5'-CTC GCT TCG GCA GCA CA-3'; R 5'-AAC GCT TCA CGA ATT TGC GT3'. human primers : miR-223-3p F 5'-ACA CTC CAG CTG GGT GTC AGT TTG TCA AAT-3'; R 5'-CTC AAC TGG TGT CGT GGA GTC GGC AAT TCA GTT GAG TGG GGT AT-3'; U6 F 5'- CGC TTC GGC AGC ACA TAT AC -3'; R 5'- AAA TAT GGA ACG CTT CAC GA -3'. Changes in gene expression levels of $>2.5$ fold were considered significant.

\section{Statistical analysis}

All data were expressed as mean \pm standard deviation (SD). Statistically significant differences among groups were evaluated by one-way analysis of variance (ANOVA) or Student's t-test using SPSS 20.0 software. Statistical significance was signified when $\mathrm{p}$ values $<0.05$.

\section{Data availability}

The data used to support the findings of this study are included within the article.

\section{Ethics approval}

The animal use protocol for this study has been reviewed and approved by the Laboratory Animal Ethical Committee, Hebei Medical University Second Affiliated Hospital.

\section{Availability of data and materials}

The datasets used and/or analyzed during the current study are available from the corresponding author on reasonable request.

\section{AUTHOR CONTRIBUTIONS}

Daofeng You: Writing-Original draft preparation, Data curation. Qiuge Qiao: Methodology, Western blotting. Katsushige Ono: Data curation. Mei Wei: Movatstaining. Wenyun Tan: RNA Isolation and real-time PCR. Cuihua Wang: Fluorescent In Situ Hybridization (FISH) assay. Yangong Liu: Macrophage culture and transfection. Gang Liu: Establishment of mice AS models. Mingqi Zheng: Writing- Reviewing and Editing, Funding acquisition. All authors have read and approved the content of the manuscript.

\section{CONFLICTS OF INTEREST}

The authors declare that they have no conflicts of interest.

\section{FUNDING}

This research was funded by grants Hebei Province Scientific and Technological Project (No. 19277757D), and S and T Program of Heibei (No. 19277793D), Hebei Natural Science Foundation (H2020206420) and Hebei Medical Key project (20200118).

\section{REFERENCES}

1. Chen J, Guo Y, Gui Y, Xu D. Physical exercise, gut, gut microbiota, and atherosclerotic cardiovascular diseases. Lipids Health Dis. 2018; 17:17. https://doi.org/10.1186/s12944-017-0653-9 PMID:29357881

2. Varghese JF, Patel R, Yadav UC. Novel Insights in the Metabolic Syndrome-induced Oxidative Stress and Inflammation-mediated Atherosclerosis. Curr Cardiol Rev. 2018; 14:4-14. https://doi.org/10.2174/1573403X13666171009112 $\underline{250}$ PMID:28990536

3. Grebe A, Hoss F, Latz E. NLRP3 Inflammasome and the IL-1 Pathway in Atherosclerosis. Circ Res. 2018; 122:1722-40. 
https://doi.org/10.1161/CIRCRESAHA.118.311362 PMID:29880500

4. Liuzzo G, Pedicino D, Flego D, Crea F. Inflammation and Atherothrombosis. In: Clinical Immunology (Fifth Edition), Elsevier. 2019; 935-946.e1. https://doi.org/10.1016/B978-0-7020-6896-6.00069-7

5. Wang $H$, Zhu J, Liu Z, Lv H, Lv P, Chen F, Fu J, Hou Y, Zhao R, Xu Y, Zhang Q, Pi J. Silencing of long isoforms of nuclear factor erythroid 2 like 1 primes macrophages towards M1 polarization. Free Radic Biol Med. 2018; 117:37-44.

https://doi.org/10.1016/j.freeradbiomed.2018.01.022 PMID:29421237

6. Chu F, Shi M, Zheng C, Shen D, Zhu J, Zheng X, Cui L. The roles of macrophages and microglia in multiple sclerosis and experimental autoimmune encephalomyelitis. J Neuroimmunol. 2018; 318:1-7.

https://doi.org/10.1016/j.jneuroim.2018.02.015 PMID:29606295

7. Dong J, Li J, Cui L, Wang Y, Lin J, Qu Y, Wang H. Cortisol modulates inflammatory responses in LPS-stimulated RAW264.7 cells via the NF-KB and MAPK pathways. BMC Vet Res. 2018; 14:30.

https://doi.org/10.1186/s12917-018-1360-0

PMID:29378573

8. Zhao X, Rao Y, Liang J, Lin S, Wang X, Li Z, Huang J. Silver Nanoparticle-Induced Phosphorylation of Histone H3 at Serine 10 Involves MAPK Pathways. Biomolecules. 2019; 9:78.

https://doi.org/10.3390/biom9020078

PMID:30813344

9. Becker $P M$, Verin $A D$, Booth $M A$, Liu F, Birukova $A$, Garcia JG. Differential regulation of diverse physiological responses to VEGF in pulmonary endothelial cells. Am J Physiol Lung Cell Mol Physiol. 2001; 281:L1500-11.

https://doi.org/10.1152/ajplung.2001.281.6.L1500 PMID:11704547

10. Kidger AM, Sipthorp J, Cook SJ. ERK1/2 inhibitors: New weapons to inhibit the RAS-regulated RAF-MEK1/2ERK1/2 pathway. Pharmacol Ther. 2018; 187:45-60. https://doi.org/10.1016/i.pharmthera.2018.02.007 PMID:29454854

11. Caunt CJ, Sale MJ, Smith PD, Cook SJ. MEK1 and MEK2 inhibitors and cancer therapy: the long and winding road. Nat Rev Cancer. 2015; 15:577-92. https://doi.org/10.1038/nrc4000 PMID:26399658

12. Chen $G$, Liu J, Jiang L, Ran X, He D, Li Y, Huang B, Wang W, Liu D, Fu S. Peiminine Protects Dopaminergic Neurons from Inflammation-Induced Cell Death by Inhibiting the ERK1/2 and NF-KB Signalling Pathways.
Int J Mol Sci. 2018; 19:821.

https://doi.org/10.3390/ijms19030821

PMID:29534526

13. Lee J, Lee $H$, Jang $\mathrm{S}$, Hong $\mathrm{SH}$, Kim WJ, Ryu SM, Park SM, Lee KH, Cho SJ, Yang SR. CMIT/MIT induce apoptosis and inflammation in alveolar epithelial cells through p38/JNK/ERK1/2 signaling pathway. Mol Cell Toxicol. 2019; 15:41-8. https://doi.org/10.1007/s13273-019-0005-0

14. Feinberg MW, Moore KJ. MicroRNA Regulation of Atherosclerosis. Circ Res. 2016; 118:703-20. https://doi.org/10.1161/CIRCRESAHA.115.306300 PMID:26892968

15. Li K, Chen ZT, Qin YW. [Expression profiles of microRNA related to atherosclerosis in patients with OSA]. Lin Chung Er Bi Yan Hou Tou Jing Wai Ke Za Zhi. 2019; 33:304-9. https://doi.org/10.13201/i.issn.10011781.2019.04.005 PMID:30970398

16. Parahuleva MS, Lipps C, Parviz B, Hölschermann $H$, Schieffer B, Schulz R, Euler G. MicroRNA expression profile of human advanced coronary atherosclerotic plaques. Sci Rep. 2018; 8:7823. https://doi.org/10.1038/s41598-018-25690-4 PMID:29777114

17. Ying SY, Chang DC, Lin SL. The MicroRNA. Methods Mol Biol. 2018; 1733:1-25.

https://doi.org/10.1007/978-1-4939-7601-0 1 PMID:29435919

18. Wu XQ, Dai $Y$, Yang $Y$, Huang $C$, Meng XM, Wu BM, Li J. Emerging role of microRNAs in regulating macrophage activation and polarization in immune response and inflammation. Immunology. 2016; 148:237-48. https://doi.org/10.1111/imm.12608 PMID:27005899

19. Wei $Y$, Schober A. MicroRNA regulation of macrophages in human pathologies. Cell Mol Life Sci. 2016; 73:3473-95. https://doi.org/10.1007/s00018-016-2254-6 PMID:27137182

20. Xie W, Yin $Q$, Zhang M, Li S, Chen S. Leukocyte miR$223-3 p$ is not associated with altered platelet responses to clopidogrel in patients with coronary artery disease. Zhong Nan Da Xue Xue Bao Yi Xue Ban. 2018; 43:421-7. https://doi.org/10.11817/j.issn.1672$\underline{\text { 7347.2018.04.014 PMID:29774880 }}$

21. Chen YC, Lin FY, Lin YW, Cheng SM, Chang CC, Lin RH, Chuang $\mathrm{CL}$, Sheu JS, Chen SM, Tsai CS. Platelet MicroRNA 365-3p Expression Correlates with High Ontreatment Platelet Reactivity in Coronary Artery Disease Patients. Cardiovasc Drugs Ther. 2019; 33:129-37. https://doi.org/10.1007/s10557-019-06855-3 
PMID: $\underline{30783954}$

22. Cheng N, Liu C, Li Y, Gao S, Han YC, Wang X, Du J, Zhang C. MicroRNA-223-3p promotes skeletal muscle regeneration by regulating inflammation in mice. J Biol Chem. 2020; 295:10212-23.

https://doi.org/10.1074/ibc.RA119.012263 PMID:32493731

23. Li J, Tan M, Xiang Q, Zhou Z, Yan H. Thrombin-activated platelet-derived exosomes regulate endothelial cell expression of ICAM-1 via microRNA-223 during the thrombosis-inflammation response. Thromb Res. 2017; 154:96-105.

https://doi.org/10.1016/j.thromres.2017.04.016 PMID:28460288

24. Jodder J. Regulation of pri-MIRNA processing: mechanistic insights into the miRNA homeostasis in plant. Plant Cell Rep. 2021; 40:783-98. https://doi.org/10.1007/s00299-020-02660-7 PMID:33454802

25. Gharibi S, Moghimi B, Tahoori MT, Mahmudi MB, Shahvazian E, Farashahi Yazd E. The miR-223: An inflammatory MicroRNA Involved in Pathogenesis of Multiple Sclerosis. International Journal of Medical Laboratory. 2018; 5.

https://doi.org/10.18502/ijml.v5i4.152

26. Gaudet AD, Fonken LK, Watkins LR, Nelson RJ, Popovich PG. MicroRNAs: Roles in Regulating Neuroinflammation. Neuroscientist. 2018; 24:221-45. https://doi.org/10.1177/1073858417721150 PMID:28737113

27. Guo YJ, Pan WW, Liu SB, Shen ZF, Xu Y, Hu LL. ERK/MAPK signalling pathway and tumorigenesis. Exp Ther Med. 2020; 19:1997-2007. https://doi.org/10.3892/etm.2020.8454 PMID: $\underline{32104259}$

28. Jonsson $R$, Brokstad KA, Jonsson MV, Delaleu $N$, Skarstein K. Current concepts on Sjögren's syndrome classification criteria and biomarkers. Eur J Oral Sci. 2018 (Suppl 1); 126:37-48. https://doi.org/10.1111/eos.12536 PMID:30178554

29. Liu SL, Li YH, Shi GY, Chen YH, Huang CW, Hong JS, Wu HL. A novel inhibitory effect of naloxone on macrophage activation and atherosclerosis formation in mice. J Am Coll Cardiol. 2006; 48:1871-9. https://doi.org/10.1016/i.jacc.2006.07.036 PMID:17084265

30. Chistiakov DA, Melnichenko AA, Myasoedova VA, Grechko AV, Orekhov AN. Role of lipids and intraplaque hypoxia in the formation of neovascularization in atherosclerosis. Ann Med. 2017; 49:661-77. https://doi.org/10.1080/07853890.2017.1366041 PMID:28797175
31. Zhu J, Chen T, Yang L, Li Z, Wong MM, Zheng X, Pan X, Zhang $L$, Yan $H$. Regulation of microRNA-155 in atherosclerotic inflammatory responses by targeting MAP3K10. PLoS One. 2012; 7:e46551.

https://doi.org/10.1371/journal.pone.0046551 PMID:23189122

32. Tian GP, Tang YY, He PP, Lv YC, Ouyang XP, Zhao GJ, Tang SL, Wu JF, Wang JL, Peng J, Zhang M, Li Y, Cayabyab FS, et al. The effects of miR-467b on lipoprotein lipase (LPL) expression, pro-inflammatory cytokine, lipid levels and atherosclerotic lesions in apolipoprotein E knockout mice. Biochem Biophys Res Commun. 2014; 443:428-34. https://doi.org/10.1016/i.bbrc.2013.11.109 PMID:24309104

33. Ediriweera $\mathrm{H}$. Therapeutic targeting of miR-33 in atherosclerosis. Doctoral dissertation, New York University. 2014.

34. Wang LIA, Zhao HOC, Dong QIA, Wu ZEY, Zhou LEI, Zhang YAN. FP06-MO-01 Retrospective study of the relevant risk factors in the cerebral infarction patients with intra/extra-cranial artherosclerosis. J Neurol Sci. 2010; 285:S65-5. https://doi.org/10.1016/S0022-510X(09)70290-4

35. Dahl A, Lund C, Russell D. [Atherosclerosis and cerebral infarction]. Tidsskr Nor Laegeforen. 2007; 127:892-6. PMID:17435812

36. Sanon S, Dao T, Sanon VP, Chilton R. Imaging of vulnerable plaques using near-infrared spectroscopy for risk stratification of atherosclerosis. Curr Atheroscler Rep. 2013; 15:304. https://doi.org/10.1007/s11883-012-0304-6 PMID:23299644

37. Lee MS, Kwon HJ, Kim HS. Macrophages from nonobese diabetic mouse have a selective defect in IFN- $\gamma$ but not IFN- $\alpha / \beta$ receptor pathway. J Clin Immunol. 2012; 32:753-61. https://doi.org/10.1007/s10875-012-9682-3 PMID:22396045

38. Chernykh ER, Shevela YE, Sakhno LV, Tikhonova MA, Petrovsky $\mathrm{YL}$, Ostanin AA. The generation and properties of human M2-like macrophages: potential candidates for CNS repair? Cell Ther Transplant. 2010; 2:e.000080.01.

https://doi.org/10.3205/ctt-2010-en-000080.01

39. Chen WN, Guo SN, Wang JY, Jia LQ, Li DY, Tian Y. [Correlation between autophagy and polarization of macrophages in atherosclerosis plaque in arteriosclerosis obliterans amputees]. Yao Xue Xue Bao. 2016; 51:68-74. 
https://doi.org/10.3866/PKU.DXHX201506003

PMID:27405164

40. Seo JW, Baek H, Mahakian LM, Kusunose J, Hamzah J, Ruoslahti E, Ferrara KW. (64)Cu-labeled LyP-1dendrimer for PET-CT imaging of atherosclerotic plaque. Bioconjug Chem. 2014; 25:231-9.

https://doi.org/10.1021/bc400347s

PMID:24433095

41. Schwanekamp JA, Lorts A, Vagnozzi RJ, Vanhoutte D, Molkentin JD. Deletion of Periostin Protects Against Atherosclerosis in Mice by Altering Inflammation and Extracellular Matrix Remodeling. Arterioscler Thromb Vasc Biol. 2016; 36:60-8.

https://doi.org/10.1161/ATVBAHA.115.306397

PMID:26564821

42. Zhang N, Fu L, Bu Y, Yao Y, Wang Y. Downregulated expression of miR-223 promotes Toll-like receptoractivated inflammatory responses in macrophages by targeting RhoB. Mol Immunol. 2017; 91:42-8. https://doi.org/10.1016/j.molimm.2017.08.026

PMID:28881218

43. Qin L, Yang YB, Yang YX, Zhu N, Li SX, Liao DF, Zheng XL. Anti-inflammatory activity of ezetimibe by regulating NF-KB/MAPK pathway in THP-1 macrophages. Pharmacology. 2014; 93:69-75. https://doi.org/10.1159/000357953 PMID:24557496

44. Xing Z, Cardona CJ, Anunciacion J, Adams S, Dao N. Roles of the ERK MAPK in the regulation of proinflammatory and apoptotic responses in chicken macrophages infected with H9N2 avian influenza virus. J Gen Virol. 2010; 91:343-51. https://doi.org/10.1099/vir.0.015578-0 PMID: 19864500 